\title{
Tuning stimulated emission of organic thin films by molecular reorientation
}

\author{
Hao-Wu Lin, Chun-Liang Lin, and Chung-Chih $\mathrm{Wu}^{\mathrm{a})}$ \\ Department of Electrical Engineering, Graduate Institute of Electro-Optical Engineering and \\ Graduate Institute of Electronics Engineering, National Taiwan University, Taipei, Taiwan 106, \\ Republic of China \\ Teng-Chih Chao and Ken-Tsung Wong \\ Department of Chemistry, National Taiwan University, Taipei, Taiwan 106, Republic of China
}

(Received 5 May 2005; accepted 29 June 2005; published online 10 August 2005)

\begin{abstract}
In this letter, employing the spectroscopic ellipsometry, we study the phenomena of molecular reorientation in amorphous thin films of a ter(9,9-diarylfluorene) compound T3 upon annealing around or above its glass transition temperature. Molecular reorientation results in changes in the in-plane optical constants, which in turn could alter the waveguiding characteristics of a thin-film slab waveguide. Employing such properties, we demonstrate continuous tuning of the wavelength of amplified spontaneous emission of a T3 slab waveguide within one sample. Such an approach has the particular advantage of permitting facile tuning of stimulated emission after device fabrication. (c) 2005 American Institute of Physics. [DOI: 10.1063/1.2031940]
\end{abstract}

Since observations of stimulated emission (SE) in luminescent and semiconducting organic thin films, ${ }^{1-4}$ they have been extensively explored as a new class of gain media for electro-optical applications. They have various attractive characteristics, including high photoluminescence (PL) efficiency, ease of processing, versatility in substrates, and compatibility in integration with other optoelectronic devices. ${ }^{1-4}$ By varying the composition of organic films, stimulated emission [in the form of either amplified spontaneous emission (ASE) or lasing] has been demonstrated at wavelengths spanning the whole visible spectrum with low excitation thresholds. ${ }^{1-4}$ Due to generally wide gain spectra of luminescent organic semiconductors, ${ }^{5,6}$ they also provide tunability in SE wavelengths $\left(\lambda_{\mathrm{SE}}\right)$ with varying the resonating structures such as distributed feedback gratings, distributed Bragg reflectors, and external cavities etc., ${ }^{2,7}$ These methods, however, in general involve more complicated fabrication or setup.

When SE is induced in slab waveguides, one facile way of tuning $\lambda_{\mathrm{SE}}$ within the gain spectrum of a luminescent organic semiconductor is to control the types of guided modes and corresponding cutoff wavelengths through film thickness. ${ }^{1,8,9}$ When the organic film is thick enough, the slab waveguide supports guided modes for the whole emission spectrum; the $\lambda_{\mathrm{SE}}$ is thus often pinned at the one having the largest material gain. Yet, when one makes the film thin enough, only the fundamental TE (transverse electric) mode can be supported and its cutoff wavelength $\left(\lambda_{\text {cutoff }}\right)$ drops below the wavelength having the largest material gain. SE is then forced to occur at the wavelength having the largest net gain $\left(\leqslant \lambda_{\text {cutoff }}\right)$, which usually shifts with $\lambda_{\text {cutoff. }}$ Thus by moving the $\lambda_{\text {cutoff }}$ with the film thickness, one in principle can tune $\lambda_{\mathrm{SE}}$. However, with such an approach, $\lambda_{\mathrm{SE}}$ is fixed once a device is fabricated, not tunable after fabrication.

Since $\lambda_{\text {cutoff }}$ depends on both the thickness and the refractive index, the alternative way of controlling $\lambda_{\text {cutoff }}$, and thus $\lambda_{\mathrm{SE}}$ of an organic semiconductor slab waveguide, is by

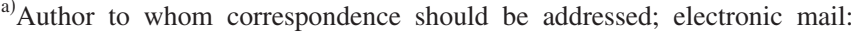
chungwu@cc.ee.ntu.edu.tw varying its refractive index. In this letter, we demonstrate a way of tuning $\lambda_{\mathrm{SE}}$ of an organic semiconductor film by varying the refractive index seen by the guided mode. Varying the refractive index of an organic film is made possible with reorientation of molecules at elevated temperatures. Molecular reorientation can be performed after devices are made, thus giving one the freedom to set or tune $\lambda_{\mathrm{SE}}$ to meet a particular purpose with a common structure.

The inset of Fig. 1 shows the structure of the material studied in this paper, a terfluorene T3 with two aryl (tolyl) substitutions on the $\mathrm{sp}^{3}$ C9 atoms. In our previous studies, ${ }^{10,11}$ we have found T3 to be a very efficient deepblue emitter in both solutions and vacuum-deposited thin films. The thin-film PL quantum yield of T3 is up to $90 \%$ and it had been applied in blue organic light-emitting devices as the emitting layer, exhibiting promising device characteristics. The introduction of rigid aryl substitutions on C9 renders the molecular structure rather steric and bulky. As a consequence, $\mathrm{T} 3$ possesses a high glass transition temperature $(\mathrm{Tg})$ of $\sim 200^{\circ} \mathrm{C}$, as shown in the trace of the differential scanning calorimetry (DSC; see Fig. 1). Thus vacuumdeposited films of T3 are homogeneous and amorphous as confirmed by x-ray diffraction, and stay morphologically stable well over $100{ }^{\circ} \mathrm{C}$. Interestingly, above $\mathrm{Tg}, \mathrm{T} 3$ does not show any sign of phase transitions (e.g., crystallization and melting, etc.) up to $400{ }^{\circ} \mathrm{C}$, indicating it be a truly amor-

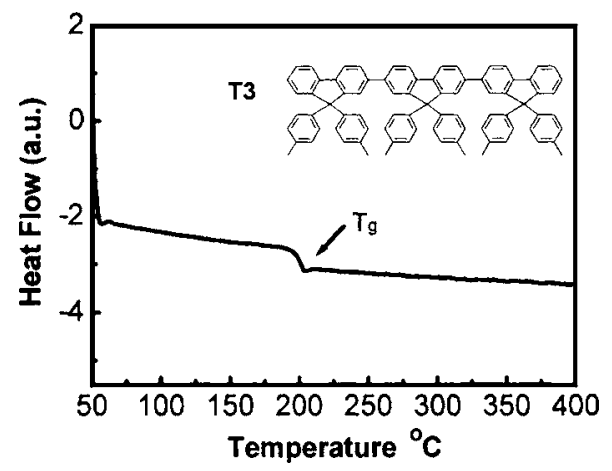

FIG. 1. The DSC trace of T3. Inset: the molecular structure of T3. 

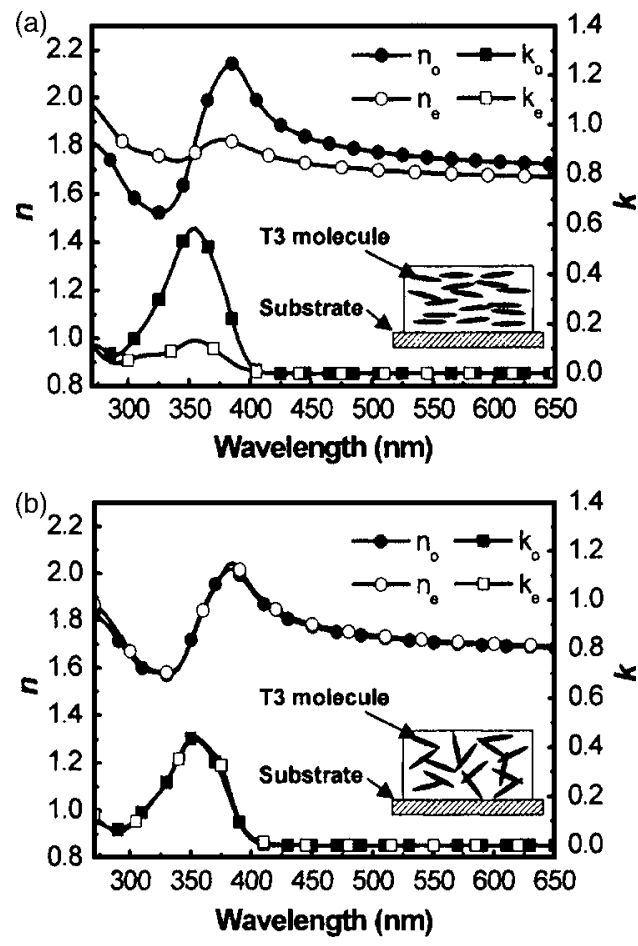

FIG. 2. Ordinary (in-plane) and extraordinary (out-of-plane) optical constants of (a) the as-deposited T3 film, and (b) the annealed T3 film $\left(200{ }^{\circ} \mathrm{C}\right.$, $4 \mathrm{~min}$ ). Insets of (a) and (b): schematic distributions of molecular orientation in the as-deposited and the annealed films.

phous material and be difficult to crystallize. Indeed, heating a T3 film at a temperature close to or well over its Tg also does not induce apparent morphological changes that can be distinguished by optical microscopy. Still, one wonders whether anything happens to T3 films above Tg and whether such properties are of any use.

Further characterization of optical constants of T3 films with spectroscopic ellipsometry reveals that annealing at temperatures close to or above its $\mathrm{Tg}$ causes significant changes in the microscopic morphology. Vacuum-deposited films of T3, in general, show optical anisotropy. Due to the high degree of correlation between model parameters for optically anisotropic films, more ellipsometric data other than those from typical reflection ellipsometry at variable angles are required to reduce correlation and to obtain correct results. $^{12,13}$ Typical approaches include taking data from samples of different thicknesses (i.e., multi-sample analysis) or combining data from both reflection and transmission ellipsometry of a same sample. ${ }^{12,13}$ In this work, anisotropic optical constants of a sample were determined by the latter approach as described by Ref. 13, which in principle is simpler and eliminates the risk of sample-to-sample variation. With this method, optical constants of T3 films obtained well-fitted the experimental ellipsometric data with low mean-squared errors (MSE) of $\leqslant 2$. Figure 2 shows spectra of the optical constants extracted for a T3 film as deposited on the fused silica substrate [Fig. 2(a)] and after subsequent annealing [Fig. 2(b)]. The annealing was performed at $200{ }^{\circ} \mathrm{C}$ for 4 min under a dry nitrogen atmosphere, and further annealing induced no more difference in optical constants. After annealing, the sample was then cooled by contact with a metal block held at the room temperature. The as-deposited film show strong uniaxial anisotropy with the optical axis along the surface normal. That is, the optical Downloaded $17 \mathrm{Feb} 2009$ to 140.112.113.225. Redistribution subje

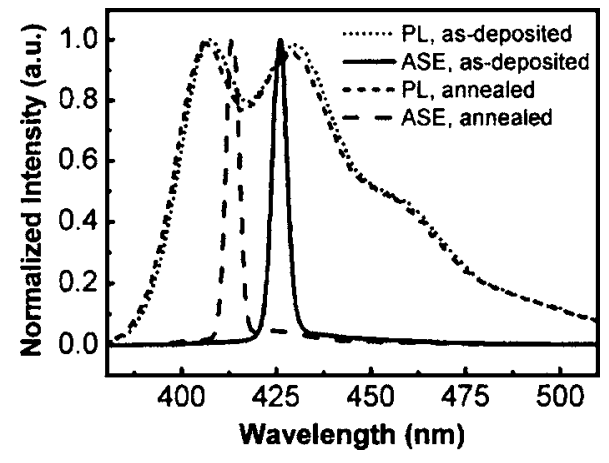

FIG. 3. PL spectra and ASE spectra of a 47-nm-thick T3 film measured as deposited and after annealing $\left(200^{\circ} \mathrm{C}, 4 \mathrm{~min}\right)$.

constants are distinguished by those for the ordinary (inplane) polarization, $n_{0}+i k_{0}$, and those for the extraordinary (out-of-plane) polarization, $n_{e}+i k_{e}$. The large difference between $k_{0}$ and $k_{e}(\sim 4.4: 1$ at the peak wavelength) indicates preferential orientations of vacuum-deposited T3 molecules parallel to the surface plane (inset of Fig. 2(a)), since the transition dipole moment for the $\pi-\pi^{*}$ transition of oligofluorenes is roughly along the backbone. ${ }^{14}$ In contrast, after annealing, the difference between in-plane and out-of-plane optical constants diminishes and the film becomes optically isotropic. The alteration of optical constants is not due to degradation of materials with annealing, since the PL spectra before and after annealing are basically the same (see Fig. 3) and the variation of PL quantum yields as determined with an integrating sphere system is within the experimental error. ${ }^{10}$ Instead, this result suggests that $\mathrm{T} 3$ molecules reorient themselves during annealing and eventually take an isotropic orientational distribution (inset of Fig. 2(a)), which is then frozen down to the room temperature with the cooling performed. Such a phenomenon shall not be too surprising since T3 is an organic glass. For organic glasses, although there occur no major morphological change or phase transition across $\mathrm{Tg}$, the viscosity in general would substantially decrease and molecules acquire larger freedom in motion and rotation. ${ }^{15}$ Characteristics unique to $\mathrm{T} 3$ here, however, are its anisotropy as deposited and its immunity to crystallization above Tg.

As a consequence of molecular reorientation, the inplane refractive index $n_{o}$ decreases by $0.06-0.1$ at wavelengths of interest (i.e., $400-450 \mathrm{~nm}$ ), which in turn would reduce $\lambda_{\text {cutoff }}$ of the silica-organic-air asymmetric slab waveguide. To demonstrate that these effects can be employed to tune $\lambda_{\mathrm{SE}}$ of the organic film, we performed ASE experiments on a 47-nm-thick T3 film deposited on fused silica. This thickness was chosen so that within the emission wavelengths of T3, only the fundamental TE mode could be supported and that its $\lambda_{\text {cutoff }}$ is set to make $\lambda_{\mathrm{SE}}$ of the asdeposited film slightly lower than the wavelength of maximal material gain (i.e., the ASE wavelength of thick films at $\sim 430 \mathrm{~nm}$ ). The third harmonic of a pulsed Nd-YAG laser $(355 \mathrm{~nm}, 10 \mathrm{~ns})$ was used as the excitation source for ASE measurements. A cylindrical lens was used to shape the laser beam into a stripe $200-\mu \mathrm{m}$-wide on the film, creating a stripe-shaped gain region. In ASE, the spontaneously emitted photons waveguided along the stripe are amplified via stimulated emission as the pump intensity is beyond a certain threshold value. As ASE occurs, most of the light is emitted from the end of the stripe and ASE manifests itself with a to AlP license or copyright; see http://apl.aip.org/apl/copyright.jsp 


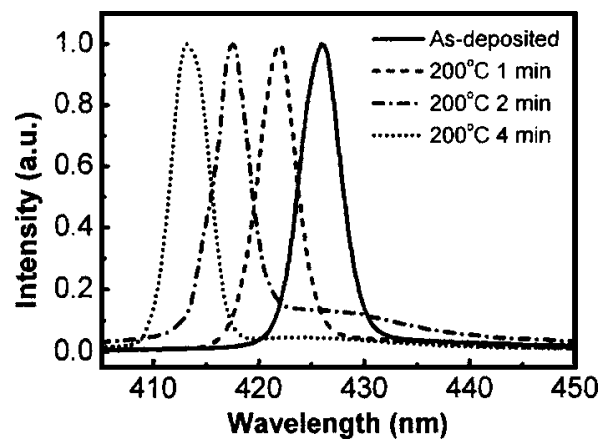

FIG. 4. ASE spectra of a 47-nm-thick T3 film measured as deposited and after different annealing conditions $\left(200^{\circ} \mathrm{C}, 1,2\right.$ and $\left.4 \mathrm{~min}\right)$.

sudden narrowing of the emission spectrum and an increase in the intensity of edge emission. The edge emission in the plane of the film was detected at one end of the pumping stripe using a fiber bundle connected to a cooled chargecoupled device (CCD) spectrograph.

Figure 3 shows the normalized PL spectra and edgecollected ASE spectra (well above the ASE threshold) from a same sample measured as deposited and after subsequent annealing $\left(200{ }^{\circ} \mathrm{C}, 4 \mathrm{~min}\right)$. The ASE of the as-deposited film occurs at $426 \mathrm{~nm}$ with a full width at half maxima (FWHM) of $\sim 4.6 \mathrm{~nm}$, while after annealing ASE switches to $413 \mathrm{~nm}$ with a FWHM of also $\sim 4.6 \mathrm{~nm}$. Although the PL spectra are basically the same in both cases, there is a 13-nm blue shift in the ASE wavelength after annealing. This shift of the ASE wavelength is consistent with the blue shift of $\lambda_{\text {cutoff }}$ of the asymmetric waveguide associated with the reorientationinduced reduction of the in-plane index $n_{o}$.

In testing different annealing conditions, we had found that the molecular reorientation is not instant for annealing temperatures around Tg of T3. Such properties render possible fine control of the distribution of molecular orientations from highly anisotropic to fully isotropic by simply adjusting the annealing temperature and the annealing time. Consequently, one may continuously tune the in-plane refractive index $n_{o}, \lambda_{\text {cutoff }}$, and the ASE wavelength between those of the two extreme situations. To demonstrate this capability of continuous tuning within one sample, we performed repeated annealing-cooling-ASE testing cycles on one 47-nm sample. Figure 4 shows the ASE spectra from one same sample taken as deposited and after annealing at $200{ }^{\circ} \mathrm{C}$ for 1,2 , and 4 min. Continuous blue shift of the ASE wavelength from $426 \mathrm{~nm}$ to $413 \mathrm{~nm}$ is observed, demonstrating the capability of continuous tuning of the ASE wavelengths with fine control of molecular reorientation.

In summary, we have shown that in the solid film of a terfluorene compound T3, molecules can reorient themselves upon annealing around or above Tg. Molecular reorientation alters the in-plane optical constants. Employing such properties, we have demonstrated continuous tuning of the ASE wavelength of a T3 slab waveguide within one sample. Such an approach has the particular advantage of permitting facile tuning of stimulated emission after device fabrication.

The authors would like to acknowledge financial support from National Science Council of Republic of China. C.-L.L. is also grateful for financial support from MediaTek Fellowship.

${ }^{1}$ F. Hide, M. A. Diáz-Garcia, B. J. Schwartz, M. R. Andersson, Q. Pei, and A. J. Heeger, Science 273, 1833 (1996).

${ }^{2}$ N. Tessler, G. J. Denton, and R. H. Friend, Nature (London) 382, 695 (1996).

${ }^{3}$ V. G. Kozlov, V. Bulovic, P. E. Burrows, and S. R. Forrest, Nature (London) 389, 362 (1997).

${ }^{4}$ M. Berggren, A. Dodabalapur, R. E. Slusher, and Z. Bao, Nature (London) 389, 466 (1997).

${ }^{5}$ G. J. Denton, N. Tessler, N. T. Harrison, and R. H. Friend, Phys. Rev. Lett. 78, 733 (1997).

${ }^{6}$ S. V. Frolov, M. Liess, P. A. Lane, W. Gellerman, Z. V. Vardeny, M. Ozaki, and K. Yoshino, Phys. Rev. Lett. 78, 4285 (1997).

${ }^{7}$ N. D. Kumar, J. D. Bhawalkar, P. N. Prasad, F. E. Karasz, and B. Hu, Appl. Phys. Lett. 71, 999 (1997).

${ }^{8}$ A. K. Sheridan, G. A. Turnbull, A. N. Safonov, and I. D. W. Samuel, Phys. Rev. B 62, R11 929 (2000).

${ }^{9}$ M. Pauchard, J. Swensen, D. Moses, A. J. Heeger, E. Perzon, and M. R. Andersson, J. Appl. Phys. 94, 3543 (2003).

${ }^{10}$ K.-T. Wong, Y.-Y. Chien, R.-T. Chen, C.-F. Wang, Y.-T. Lin, H.-H. Chiang, P.-Y. Hsieh, C.-C. Wu, C.-H. Chou, Y.-O. Su, G.-H. Lee, and S.-M. Peng, J. Am. Chem. Soc. 124, 11576 (2002).

${ }^{11}$ C.-C. Wu, Y.-T. Lin, K.-T. Wong, R.-T. Chen, and Y.-Y. Chien, Adv. Mater. (Weinheim, Ger.) 16, 61 (2004).

${ }^{12}$ M. Tammer and A. P. Monkman, Adv. Mater. (Weinheim, Ger.) 14, 210 (2002).

${ }^{13}$ C. M. Ramsdale and N. C. Greenham, Adv. Mater. (Weinheim, Ger.) 14, 212 (2002).

${ }^{14}$ Y. Geng, A. Trajkovska, D. Katsis, J. J. Ou, S. W. Culligan, and S. H. Chen, J. Am. Chem. Soc. 124, 8337 (2002).

${ }^{15}$ K. Naito and A. Miura, J. Phys. Chem. 97, 6240 (1993). 